। n Salamanca fanden sich im Wintersemester 2002/03 einige Interessierte (Dozenten, Studenten und andere Leser) zusammen, um einigen Texten der neuesten deutschsprachigen Literatur auf den Zahn zu fühlen.' Wir lasen Bücher bzw. Ausschnitte aus Büchern, von denen wir gehört hatten; die Preise gewonnen hatten, die mit (Kritiker-)Lob überschüttet worden waren und/oder denen Verkaufserfolge beschieden waren; die mit Etiketten wie "Popliteratur", "Fräuleinwunder" oder "Großer Wenderoman" versehen worden waren. Sie hatten eins gemeinsam: sie waren in den letzten sieben Jahren erschienen und fielen damit in die vielleicht nur chronolo-
Sturmlauf der Moderne gegen das Erzählen nie gegeben." In dieser knappen Bemerkung von Burkhard Spinnen, Klappentext der Taschenbuchausgabe von Sommerhaus, später, stecken mindestens zwei Behauptungen, deren Berechtigung gar nicht geklärt ist: erstens, dass die aktuelle jüngste Autorengeneration von sich (selbst) erzählt; und zweitens, dass sie hinter gewisse etablierte Standards der Moderne zurückkehrt; und beide Behauptungen scheinen miteinander zusammenzuhängen.

Was Spinnen hier lobend hervorhebt, ist von anderen mit umgekehrtem Vorzeichen als Vorwurf formuliert worden.

\title{
VON WEGEN EINFACH ERZÄHLT. NEUESTE DEUTSCHE PROSA
}

gisch zu benennende Gruppe der neuen deutschen Literatur der neunziger Jahre. Die Frage, die uns leitete, war ganz allgemein gehalten: Was hat es mit dieser Literatur auf sich? Oder vielleicht etwas konkreter: Lohnt sich die Lektüre? Oder noch konkreter: Was hat diese

\section{Nadja Nitsche SALAMANCA} Literatur tatsächlich mit den Urteilen und Klassifizierungen zu tun, die man inzwischen überall über sie lesen kann?

Um es vorweg zu nehmen: Die Ergebnisse unserer Lektüre, so stichprobenartig sie auch war, waren in mancher Hinsicht überraschend und vielleicht in einiger Hinsicht aufschlussreich.

\section{Wer ist eigentlich Ich?}

Judith Hermann ist nach Erscheinen ihres ersten Buches, Sommerhaus, später; als eins der'größten Nachwuchstalente gefeiert worden - bis hin zum Kleistpreis, den sie 2001 erhielt. Anfang dieses Jahres ist nach langer Wartezeit, die die Spannung nur noch steigerte, ihr zweites Buch, Nichts als Gespenster, erschienen und, wie es fast schon nicht anders zu erwarten war, völlig verrissen worden. Das lässt den Verdacht aufkommen: Vielleicht war das erste Buch nicht so gut, und vielleicht ist das zweite nicht so schlecht wie die Kritik sie gemacht hat.

"Wenn die jeweils jüngste Generation von sich schreibt, dann braucht sie dazu nicht notwendig eine ganz und gar 'neue', 'nie dagewesene' Sprache. Judith Hermanns Geschichten sind so wunderbar gelassen erzählt, als habe es den
Schon 1989 schrieb Frank Schirrmacher: "Als 'Authentizität' tarnt sich nun schon seit Jahren die Talentschwäche der nachwachsenden Schriftsteller. ${ }^{2 \prime}$ Er eröffnete mit dieser Polemik eine Debatte über den Stand der deutschsprachigen Literatur, in der sich zwei Fraktionen abzeichneten: Die eine verlangte eine gegenwärtige Literatur, die Geschichten erzählt, sich Spannung, Unterhaltung und Plot zutraut und auf die Problematisierungen des Erzählens verzichtet, die so viel (deutschsprachige) Literatur (des 20. Jahrhunderts) so schwer verdaulich machten. Einfach erzählen!, war die Forderung. Die andere Fraktion verwarf - wie Schirrmacher - (fast) alle Gegenwartliteratur wegen Oberflächlichkeit, Unterhaltungssucht, Langeweile und falsch verstandener oder falsch verwendeter Authentizität.

Judith Hermann scheint im Guten wie im Schlechten diesen Kriterien zu entsprechen. Jung, photogen, mit interessanter, ein bisschen abenteuerlicher Biographie, schreibt sie Geschichten, in denen junge, vermutlich gutaussehende, ein bisschen abenteuerlich lebende Frauen die Hauptfiguren oder gleich die Ich-Erzählerinnen sind. Oder doch nicht?

In der Titelgeschichte des Erzählungsbandes Sommerhaus, später haben alle Figuren nur Vornamen, bis auf eine[n], der nur über den allerdings vielsagenden Nachnamen Stein verfügt, obwohl er vielleicht weniger steinern ist als zum Beispiel das Ich, das in einer irgendwie speziellen 
Beziehung zu Stein steht, ohne das diese Beziehung (auf welche Art auch immer) so richtig in Gang kommen würde. Das titelgebende Sommerhaus (von denen es sogar mehrere gibt: "die schäbigen, schiefen, kleinen Landhäuschen, die sie bald alle hatten") ist für alle irgendwie wichtig, zumal die Geschichte hauptsächlich im Winter spielt; obwohl auch diese Wichtigkeit etwas diffus bleibt. Die meisten Figuren sind Künstler, aber ihre Leben und ihr Umgang miteinander scheinen weniger von Kreativität und Freiheit als von Kälte und Nichtverstehen geprägt.

Bei all diesen Klischees dürfte den meisten Lesern gar nicht auffallen, dass man in Wirklichkeit nicht mal weiß, ob man eine Ich-Erzählerin oder einen Ich-Erzähler vor sich hat. Man muss schon sehr genau hinschauen, um zu sehen, dass es in mehr als einer Geschichte des Bandes keine konkreten Hinweise weder auf das eine noch auf das andere gibt; nur Angebote zum interpretatorischen Kurzschluss, auf die man als Leser bei den ersten ein, zwei Lektüren bequem hereinfallen kann. "Ich" und "mein Geliebter" heißt es, oder: "Falk küsste Anna, und Anna küßte mich, und ich küßte Christiane", aber wer "ich" ist, wird auf diese Weise nicht geklärt.

Dieses kleine Detail sollte einem Leser doch zu denken geben. Vielleicht sind die Geschichten vor allem deshalb (überwiegend) in der ersten Person erzählt, um eben diesen Trick zu ermöglichen? Der ja über ein Spiel mit den Personalpronomen hinausgeht: Der Leser wird so nah an das erzählende lch herangeführt, dass er dieses Ich selbst nicht mehr in den Blick bekommt und nur nach außen blicken kann. Natürlich könnte es sein, dass das Ich große Ähnlichkeit mit der Autorin hat; aber diese Ähnlichkeit oder gar eine ldentität als gegeben zu nehmen ist eindeutig eine verkürzte Lesart. Und einen Leserirrtum, der auf ungenaue Lektüre zurückgeht, der Autorin zur Last zu legen, kann jedenfalls nicht angehen.

\section{Und was ist nun mit dem Dachs?}

Ingo Schulzes Simple Storys spielen schon im Titel mit den Assoziationen von "einfach": Einfache Geschichten scheint der Titel anzukündigen, und kleine Zusammenfassungen, die jedem Kapitel vorangestellt sind und die man im Inhaltsverzeichnis bequem überblicken kann, scheinen das zu bestätigen. "Lydia erzählt von Dr. Barbara Holitzschek, die behauptet, einen Dachs überfahren zu haben. Ein langes Gespräch über Tiere. Die Unfallstelle. Rätselhaftes Ende ohne Dachs", heißt es zum Beispiel über das fünfte Kapitel. Ingo Schulze selbst hat sich zur Funktion dieser Zusammenfassungen folgendermaßen geäußert: Es "befindet sich vor jedem Kapitel eine Art Stolperstein gegen die Einfühlung, eine 'Episierung' des Inhalts, die signalisiert: Ich kann das ganze auch anders erzählen, als Banalität, als simple Story, als die Zusammenfassung für einen Verriss." ${ }^{\text {"3 }}$

Andererseits stellen diese Inhaltsangaben aber auch eine Beziehung zur Gattung der Novelle her, die stets eine unerhörte Begebenheit erzählen und sich in wenigen Worten auf den Punkt bringen lassen soll. Tatsächlich sind die "Simplen Storys" ebenso alltäglich wie unerhört: Das Romanpersonal sind "einfache" Leute, also Leute, die strampeln müssen, um zu überleben. Es widerfahren ihnen alle Arten von Unglücken, aber keines, das nicht auch jedem Leser widerfahren könnte. Die Geschichten sind vielleicht einfach im Sinne von alltäglich, aber keineswegs einfach im Sinne von unkompliziert (das Leben ist halt alles andere als einfach...).

Einfach und kompliziert zugleich ist auch die Erzählweise, die an ein Drehbuch erinnert: der Leser erfährt alles, was man sehen und hören kann; aber nicht mehr. Was fehlt, sind die Erklärungen dazu, die Zusammenhänge, die sich unter der Oberfläche verbergen und die man nur entdeckt, wenn man sehr genau hinschaut: Was ist nun eigentlich mit dem Dachs aus Kapitel 6?

Im Grunde verwendet Schulze also einen ähnlichen Trick wie Hermann: eine Perspektive, die nur den Blick von außen auf die Figuren zulässt, keine Innenansichten. Natürlich ist der scheinbar so objektive Blick manipuliert. Wo bei Hermann Idiosynkrasien und Beschränkungen des erzählenden lchs das Bild färben, ist es bei Schulze der unsichtbare Erzähler, der die Kamera führt und die Bildauswahl bestimmt.

Der Unterschied liegt aber darin, dass Schulze sich auch bei oberflächlicher Lektüre mit keiner seiner Romanfiguren identifizieren lässt und - obwohl er sich anders als Hermann nicht selten auch in Essays und Vorträgen zu literarischen oder nicht-literarischen Themen äußert - kaum Leserfantasie auf seine Person zieht. Er ist also sicher vor allen Authentizitäts-Vorwürfen; und ist kuriosierweise genau dafür kritisiert worden: "Die Mehrzahl der Rezensenten [von 33 Augenblicke des Glücks, Schulzes erstem Buch; N.N.], auch der wohlmeinenden, merkten an, daß der Autor zwar hier und da gekonnt erzähle, aber er sage nirgendwo $\mathrm{ICH}$, er habe seinen Ton, seine Stimme, seinen Stil, seine Schreibweise noch nicht gefunden. [..] Auf Lesungen bekam ich zu hören: Aber wo sind Sie denn in Ihren Geschichten? Wo sagen Sie ICH? ${ }^{14}$

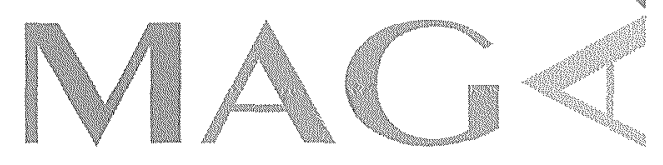




\section{"Surface is an illusion, but so is depth"}

Dieses David Hockney-Zitat hat Christian Kracht seiner Sammlung von Reisefeuilletons mit dem gut danebengegriffenen Titel Dergelbe Bleistift vorangestellt, und schickt damit seine Leser in eine Schleife: Macht sich Kracht nun darüber lustig, dass man in seinen Texten Tiefe sucht, wo nur Oberfläche ist, oder vielleicht umgekehrt darüber, dass man sich mit der Oberfläche beschäftigt, wo man doch ebensogut die (scheinbare oder echte) Tiefe erkunden könnte?

Noch wer seine Bücher lobt, scheint sie misszuverstehen (insofern kann ich für das folgende nicht garantieren...), und das scheint voll in der Absicht des Autors zu liegen, der sich bis in Lesungen, Interviews und seine Homepage hinein als die Figur inszeniert, als die er auch in seinen (stets in der ersten Person erzählten) Texten auftritt. Er schrieb mit im recht tagebuchartigen OnlineKollektivschreibexperiment pool; aber über ihn oder seinen Alltag erfuhren die Leser dabei nichts. Es kursieren reichlich Fotos von ihm, auf denen er sich auch stets sehr ähnlich sieht; aber kann man wissen, ob er es wirklich selbst ist?

Krachts Protagonisten werden als Yuppies oder Herrenmenschen wahrgenommen, die mit Designermarken und Drogen besser umgehen können als mit ihren Freunden und viel Zeit auf kostspieligen Reisen in exotische Länder verbringen, in denen sie nichts anderes zu suchen scheinen als in einer Münchner Disco oder an einer Sylter Edelfischbude. Solche Lifestyle-Details, die die Literatur von Kracht und anderen Pop-Literaten versammelt, werden von einem Teil des Leser- und KritikerPublikums als das eigentliche Verdienst dieser Literatur gewertet. So prüfte der Rostocker Literaturwissenschaftler Moritz Baßler in seiner 2002 erschienenen Untersuchung. über den "Deutschen PopRoman" mit einigem theoretischen Aufwand eine Reihe neuerer Bücher auf ihren Beitrag zum "Archiv", das die Literatur darstellt; da es in (jeder) Gegenwartsliteratur vor allem um die Gegenwart zu gehen habe, schneiden bei $\mathrm{ihm}$ diejenigen Autoren am besten ab, die am meisten Gegenwart für die Ewigkeit sichern.

Ein solches Kriterium mag seine Berechtigung haben, begrenzt aber die Relevanz von Literatur in geradezu positivistischer Weise und beantwortet vor allem eine Frage nicht, die in diesem Zusammenhang seltsamerweise nie gestellt wird. WARUM schreibt zum Beispiel Kracht zum Beispiel über teure Anzüge? Nicht, dass er solches Material für seine Literatur benutzt, ist relevant, sondern, wie (und wie gut) er das tut.
Ohne diese Frage für Kracht letztgültig beantworten zu wollen oder zu können, hier nur noch ein Hinweis darauf, dass selbst noch die Oberfläche von Krachts Texten Warnungen vor zu naiver Lektüre liefert: Als Schweizer, der er ist (worauf in allen Klappentexten speziell hingewiesen wird) müsste er in Faserland der nationalsozialistischen Vergangenheit Deutschlands nicht so viel Raum einräumen, wie er es tatsächlich tut. Dieses Detail liegt quer zur Wahrnehmung des Buches als Lifestyle- und Lebensgefühl-Literatur, und wird (wahrscheinlich deshalb) gerne komplett überlesen.

\section{Wenn ein Kopf und ein Buch zusammenstoßen...}

Kracht, Schulze und Hermann werden normalerweise nicht in dieselbe Kiste geräumt, aber sie haben eins gemeinsam: sie verstehen ihr Handwerk - vielleicht der als Pop- und Event-Literat aussortierte Kracht sogar besser als die Kleist-Preis-geehrte Hermann. Woher kommen aber die im positiven wie im negativen seltsam überzogenen Reaktionen des Literaturbetriebs? Warum schreibt man Autoren wie diesen drei die Rettung der deutschen Literatur und fast gleichzeitig schon wieder den letzten Beitrag zu ihrem endgültigen Untergang zu? ${ }^{5}$

Eine Erklärung könnte in einer Lieblingsbeschäftigung des Betriebs vor den $90 \mathrm{er}$ Jahren (und seither nicht minder) liegen: dem lauthalsen Lamentieren über literarische Langeweile und - nicht immer, aber immer öfter - den Rufen nach neuer, unkomplizierter, komsumierbarer deutschsprachiger Literatur. Der Großkritiker Marcel Reich-Ranicki, die wahrscheinlich einflussreichste Einzelgestalt des Betriebs, predigte jahrelang in seinem "Literarischen Quartett" das Vorbild der (spannenden, unterhaltsamen, erzählfreudigen) US-amerikanischen Literatur. Und Urteile wie das zitierte über Hermann, die das "gelassene", von der Moderne unangekränkelte Erzählen loben, entsprechen doch auf verblüffende Weise dieser Forderung.

Nun berufen sich alle drei vorgestellten Autoren explizit oder implizit auf nordamerikanische literarische Traditionen und Vorbilder. Schulze pflegt die Kunst der wortkargen und vielsagenden Dialoge, die er aus den amerikanischen Short Stories gelernt hat; der ebenso kultige wie schwerverdauliche Bret Easton Ellis lässt sich 


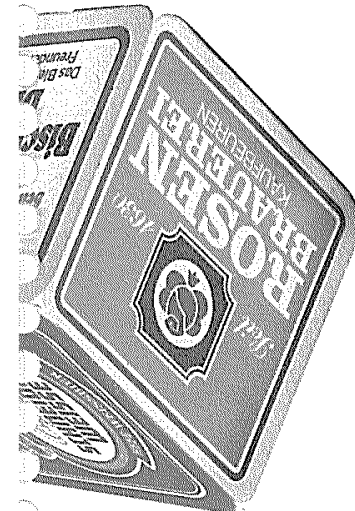

unschwer als Referenz für Krachts Literatur ausmachen; Hermann benennt einen ihrer Protagonisten nach dem amerikanischen Journalisten und Kult-Autor Hunter S. Thompson. Aber heißt das zwangsläufig, dass die deutschen Autoren hinter dem literarischen Anspruch ihrer Vorbilder zurückbleiben?

Weil die Kritik so lange nach "unterhaltender" Literatur verlangt hat, glaubte sie in der irgendwie neuen, irgendwie anderen Literatur junger Autoren der $90 \mathrm{er}$ Jahre das zu finden, was sie zu suchen glaubte: unkomplizierte, konsumierbare Literatur mit klaren Identifikationsangeboten und ohne theoretischen Ballast. - Um dann ziemlich schnell am eigenen Leser-Leib zu erfahren, dass das bloß Unterhaltende (und eben auch alles andere, wenn man es bloß unter derart definierten Unterhaltungsgesichtspunkten liest) gar nicht unterhält, sondern ziemlich schnell langweilt.

Nicht die Literatur ist plötzlich naiver geworden; die Kritik hat sich aus kaum verständlichen Gründen plötzlich entschlossen, einfältiger zu lesen. Wie Jochen Hörisch frei nach Lichtenberg formuliert hat: Es gibt schlechte Bücher, es gibt aber auch dumme Lektüren. ${ }^{6}$

\section{Literaturliste}

Im folgenden einige Tips für eigene, hoffentlich nicht dumme Lektüren:

- Julia Franck: Bauchlandung. Geschichten zum Anfassen, Köln (DuMont) 2000.
- Judith Hermann: Sommerhaus, später. Erzählungen Frankfurt a.M. (Fischer) 1998.

- Judith Hermann: Nichts als Gespenster, Frankfurt a.M (S. Fischer) 2003.

- Georg Klein: Barbar Rosa. Eine Detektivgeschichte, Berlin (Alexander Fest) 2001.

- Christian Kracht: Faserland, Köln (Kiepenheuer \& Witsch) 1995.

- Christian Kracht: Der gelbe Bleistift, Köln (Kiepenheuer \& Witsch) 2000.

- Andreas Maier: Wäldchestag, Frankfurt a.M. (Suhrkamp) 2000. - pool <http://home.snafu.de/ampool/index2.htm>.

- Ingo Schulze: 33 Augenblicke des Glücks, Berlin (Berlin Verlag) 1995

- Ingo Schulze: Simple Storys. Ein Roman aus der ostdeutschen Provinz, Berlin (Berlin Verlag) 1998.

\section{Kritik}

- Moritz Baßler: Der deutsche Pop-Roman. Die neuen Archivisten, München (C.H.Beck) 2002.

- Andrea Köhler, Rainer Moritz (Hgg.): Maulhelden und Königskinder. Zur Debatte über die deutschsprachige Gegenwartsliteratur, Leipzig (Reclam Leipzig) 1998.

- Ute-Christine Krupp, Ulrike Janssen (Hgg.): Zuerst bin ich immer Leser. Prosa schreiben heute, Frankfurt a.M. (edition suhrkamp) 2000.

- Frauke Meyer-Gosau: "Lob der Flaute. Endlich tun Debütanten wieder, was sie können: ganz Verschiedenes", in: Literaturen 1/2 2003, S.79-81.

- Rainer Moritz: "Die Scheinblüte. Vom neuerlichen Desinteresse an deutschsprachiger Literatur", in: Literaturen 1/2 2003, S.76-78.

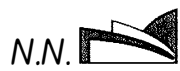

\section{NOTAS}

1. Wir lasen: Ingo Schulze: Simple Storys; Christian Kracht: Faserland; Judith Hermann: Sommerhaus, später, Julia Franck: Mir nichts, dir nichts; Andreas Maier: Wäldchestag; Georg Klein: Barbar Rosa; siehe Literaturliste.

2. Frank Schirrmacher, "Idyllen in der Wüste oder Das Versagen vor der Metropole. Überlebenstechniken der jungen deutschen Literatur am Ende der achtziger Jahre", in: Andrea Köhler, Rainer Moritz (Hgg.), Maulhelden und Königskinder. Zur Debatte über die deutschsprachige Gegenwartsliteratur, Leipzig (Reclam Leipzig) 1998, S.15-27, hier: S.21.

3. Ingo Schulze: "Lesen und Schreiben"; in: Ute-Christine Krupp, Ulrike Janssen (Hgg.), Zuerst bin ich immer Leser. Prosa schreiben heute, Frankfurt a.M. (edition suhrkamp) 2000, S.80-101, hier: S.96.

4. Ebd. S.92.

5. Das Literaturen-Heft vom Januar/Februar 2003 brachte unter dem Stichwort "Debütanten" der Einfachheit halber gleich zu beiden Standpunkten je einen Beitrag: Rainer Moritz: "Die Scheinblüte. Vom neuerlichen Desinteresse an deutschsprachiger Literatur", S.76-78, und Frauke Meyer-Gosau: "Lob der Flaute. Endlich tun Debütanten wieder, was sie können: ganz Verschiedenes", S.79-81.

6. Jochen Hörisch: "Die Vorzüge der Gegenwartsliteratur", in: Andrea Köhler, Rainer Moritz (Hgg.), Maulhelden und Königskinder. Zur Debatte über die deutschsprachige Gegenwartsliteratur, Leipzig (Reclam Leipzig) 1998, S.224-231, hier: S.230.

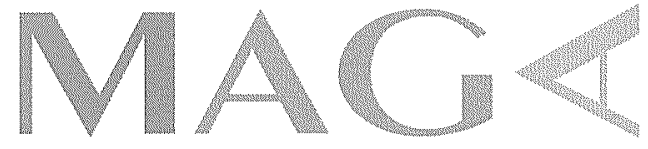

